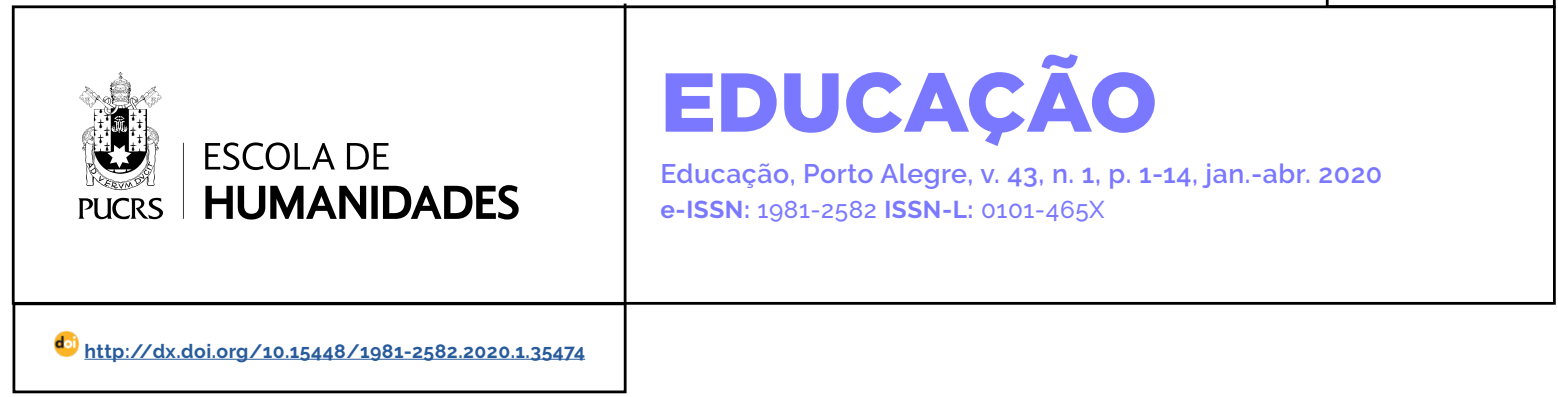

DOSSIÊ

\title{
Filtros mentales, coaching y Atención Temprana centrada en la familia
}

\author{
Mental filters, coaching and Family-Centered Early Intervention \\ Filtros Mentais, coaching e Intervenção Precoce centrada na familia
}

\section{Noelia Orcajada \\ Sánchez ${ }^{1}$}

orcid.org/0000-0003-3476-9291

noelia.orcajada@um.es

\section{Francisco Alberto \\ Garcia Sánchez ${ }^{1}$}

orcid.org/0000-0003-3788-6354

fags@um.es

Recebido em: 31 ago. 2019.

Aprovado em: 20 nov. 2019

Publicado em: 24 mai, 2020.
Resumen: El profesional de Atención Temprana que implementa unas Prácticas Centradas en la Familia, lo hace convencido de los buenos resultados de estas prácticas. De ello dejan evidencia tanto estudios originales como trabajos de revisión sistemática y meta-análisis. Ahora bien, el profesional siempre presenta dudas en torno a su propia práctica y a su capacidad para alcanzar el nivel de implicación que quiere de las familias. Entender la interpretación del adulto sobre su realidad y sus competencias, ayuda al profesional a ponerse en el lugar de las familias, para conseguir su implicación desde su perspectiva y lenguaje propios. En este artículo abordaremos la identificación y comprensión de los filtros del adulto. Esto se hará dentro de unas estrategias de coaching adaptadas a las necesidades de la Atención Temprana, que será de especial utilidad para favorecer la reflexión en las familias.

Palabras-clave: filtros mentales, coaching, Atención Temprana

Resumo: O profissional de Intervenção Precoce que implementa práticas centradas na família o faz convencido dos bons resultados dessas práticas. Para tanto, busca evidências tanto em estudos originais, quanto em revisões sistemáticas e de meta-análise. No entanto, o profissional sempre apresenta dúvidas sobre sua própria prática e sua capacidade de atingir o nivel de envolvimento que deseja das familias. Compreender a interpretação que o adulto faz sobre sua realidade e suas competências ajuda o profissional a se colocar no lugar da familia, de modo a conseguir seu envolvimento a partir de sua própria perspectiva e linguagem. Neste artigo, abordaremos a identificação e o entendimento dos filtros do adulto. Isso será feito dentro de estratégias de treinamento adaptadas às necessidades da Intervenção Precoce, que serão especialmente úteis para incentivar a reflexão nas familias.

Palavras-chave: filtros mentais, coaching, Intervenção Precoce

Abstract: The Early Intervention professional who implements Family-Centered Practices, does it convinced of the good outcomes one can obtain as result of using these practices. This is evidenced-based either through original studies and systematic review as well as meta-analysis. However, the professional always has doubts about his own practice and his ability to reach the level of involvement he wants from families. Understanding the adult's interpretation of his reality and his competences, helps the professional to put himself in the place of the families, to get their involvement from their own perspective and language. In this paper we will address the identification and understanding of adult filters. This will be done within coaching strategies adapted to the needs of Early Intervention, which will be especially useful to encourage reflection in the families.

Keywords: mental filters, coaching, Early Intervention 


\section{INTRODUCCIÓN}

En Atención Temprana (AT) siempre se ha destacado el papel fundamental de la familia en el desarrollo de su hijo. Sin embargo, son las Prácticas de intervención Centradas en la Familia (PCF), las que les han otorgado el protagonismo que merecen: haciéndolas partícipes de las decisiones a tomar, escuchando sus necesidades, atendiendo sus prioridades. Y, siempre, partiendo desde su propia perspectiva, respetando sus valores y creencias, gracias a lo cual el profesional consigue la confianza de la familia y su alianza para el desarrollo de su hijo. Para ello, haremos uso de todo un conjunto de prácticas relacionales y participativas (Dunst, 2000; 2002). Pero también utilizaremos otras herramientas, como el coaching, para favorecer la reflexión de los cuidadores principales de cara al aprovechamiento de las oportunidades de aprendizaje que oferta su entorno y sus rutinas.

Ahora bien, entender y profundizar en el concepto de coaching nos lleva a entender que no es suficiente con la labor que el profesional haga para favorecer esa reflexión, las conclusiones a las que llegue la familia y su nivel de implicación. Todo ello también dependerá de una serie de filtros que condicionarán la interpretación que la familia haga de su propia realidad. Por ello, es importante que el profesional conozca esos filtros y sepa identificarlos a la hora de trabajar con las familias, con el fin de adaptar su lenguaje y ajustar su discurso a las propias necesidades familiares. En este artículo abordaremos la identificación y comprensión de diferentes filtros mentales, diferentes automatismos perceptivo-cognitivos, asi como diferentes metaprogramas presentes en el adulto y que condicionan la perspectiva desde la que la familia observa su propia realidad. Todo ello se abordará a través de una revisión teórica sobre la temática y, siempre, relacionándolo con la realidad que viven familias y profesionales dentro del ámbito de la AT.

El término "coaching" no tiene una definición única debido a su procedencia diversa y a que podemos encontrar diferentes definiciones según la escuela o ámbito en que nos centremos, como el académico, el organizacional o el político (Benavides, 2014; Ortiz, 2010; Sans, 2012). Además, actualmente podemos nombrar, también, el ámbito familiar, asi como el coaching en Atención Temprana. Bisquerra (2008) apunta que el coaching se basa en una conversación en la que intervienen, al menos, dos personas. En ella, el coach no dice al coachee, o persona que recibe el coaching, lo que debe hacer, sino que lo ayuda a descubrirlo por sí mismo y a activar los recursos necesarios para conseguirlo. El coaching aporta un aprendizaje que conlleva una transformación en el coachee que perdura en el tiempo, conseguida a través de acciones y reflexiones continuas (Lozano, 2008).

Rush y Shelden (2011) definen el coaching en AT como una estrategia de aprendizaje adulto en la que el entrenador promueve la capacidad del usuario para reflexionar sobre sus acciones, como un medio para determinar la efectividad de una acción o práctica y desarrollar un plan para el uso de la acción en situaciones inmediatas y futuras. Para ello, el profesional de AT, cuenta con una serie de estrategias (Friedman, Woods \& Salisbury, 2012):

- Información bidireccional. Se da cuando el cuidador principal y el profesional intercambian información sobre un tema de interés, como puede ser la crianza, las características de la discapacidad, etc.

- Observación directa sin intervención. Dejar que los padres actúen con su hijo como lo hacen normalmente, para analizar su participación, implicación y estrategias de comunicación.

- Enseñanza directa a los padres. Enseñar estrategias específicas a los cuidadores para favorecer un determinado aprendizaje, utilizando información verbal, impresa o visual.

- Demostración de la intervención. Hacer de modelo poniendo en marcha una determinada estrategia que se va describiendo detalladamente al cuidador, dando todas las explicaciones necesarias para que pueda entenderla. 
- Práctica guiada con feedback. El profesional guía al cuidador y le da su apoyo ante una determinada estrategia que quiere poner en marcha. Para ello se propicia o busca la situación necesaria.

- Feedback sobre la práctica espontánea del cuidador. El cuidador principal interacciona con el niño de forma natural y el profesional sólo intervendrá para dar alguna orientación o para favorecer la reflexión en un momento dado.

- Interacciones conjuntas. Profesional y cuidador interaccionan por turnos con el niño, realizando, primero uno y luego el otro, la estrategia pactada.

- Reflexión y solución de problemas. Intercambio de información entre profesional y familia para analizar una situación, discutir alternativas y/o evaluar un determinado plan de acción.

El profesional de AT, sobre todo al comienzo de sus PCF, siempre presenta dudas sobre su propia práctica y su capacidad para alcanzar la implicación que quiere de las familias. En parte esto ocurre porque las estrategias que el profesional deberá poner en marcha deberán ajustarse a la situación, las necesidades y las características de cada familia. Además, el profesional deberá utilizar un lenguaje que conecte con el cuidador principal y le permita avanzar. Guarnieri y Ortiz (2010) señalan que el coach trabaja con palabras cotidianas que el cliente utiliza habitualmente dándole una determinada interpretación. Pero, ahora, el coach las rescata para darles una visión nueva y utilizarlas favoreciendo la reflexión, el cambio de perspectiva y, por tanto, acciones diferentes.

Para favorecer esa reflexión, el profesional debe entender que somos seres eminentemente lingüisticos que, a medida que crecemos, vamos acumulando experiencias y generando creencias con las que convivimos, pensando, en muchas ocasiones, que son "la realidad" (Sans, 2012). Estas creencias se convierten en filtros que nos ayudan a interpretar nuevas experiencias. De ahí la importancia que tiene, para el profesional de AT, conocer los diferentes filtros mentales que los adultos con los que va a intervenir pueden poner en marcha a la hora de interpretar su realidad. Y la única forma que tenemos de conocer esos filtros es a través del lenguaje. Por otra parte, también el lenguaje permitirá al profesional ponerse a la altura del adulto con el que trabaja y, partiendo desde sus propias perspectivas, favorecer la confianza, implicación y reflexión necesarias para dar respuesta a sus necesidades y prioridades.

Si somos capaces de identificar las creencias y valores que sustentan, en eladulto, la interpretación de su realidad y sus experiencias, podremos cambiar aquellas limitantes por otras facilitadoras que generen nuevas emociones que favorezcan la acción, empoderando así al coachee (Sans, 2012). Ahora bien, las creencias sólo son uno de los filtros que utiliza la mente humana a la hora de interpretar la realidad y afrontar las diferentes situaciones y experiencias. A continuación, profundizaremos en los filtros mentales que pueden ayudar al profesional de AT a conocer algo más al adulto con el que trabaja y, así y utilizando su propio lenguaje, favorecer su reflexión.

\section{FILTROS MENTALES}

Todos nos enfrentamos a diferentes problemas a lo largo de su vida y tomamos decisiones. Algunas de estas decisiones serán más difíciles que otras, tanto más cuando hay un niño con discapacidad en la familia. Es entonces cuando los cuidadores principales pierden la sensación de autocompetencia, algo que en AT debemos contribuir a recuperar, con el fin de que, como padres, vuelvan a tomar sus propias decisiones. Para ello, el profesional necesita un compromiso por parte del cuidador, para plantear objetivos que darán respuesta a sus propias necesidades y prioridades.

En unas PCF, el profesional debe tener muy claro que es la familia quien debe tomar sus propias decisiones y él ser un apoyo efectivo para ayudarla a recorrer el camino que desean para dar respuesta a sus necesidades. Sin embargo, a veces se hace difícil conseguir esa efectividad. A la hora de servir de apoyo efectivo a las familias, el profesional debe tener en cuenta que los diferentes cuidadores principales con los que 
trabaja, a lo largo de sus vidas, van teniendo diferentes experiencias. Estas marcan su interpretación de la realidad y les hace enfocarse en unos datos y no en otros. En este sentido, siempre debemos considerar que el afrontar un problema y tomar una decisión está mediado por el modo en que vemos las cosas. Para ello la mente funciona con ciertos filtros que tamizan la información que recibimos (Espíndola, 2005). Si somos capaces de identificar y valorar las creencias que nos limitan a la hora de conseguir nuestros objetivos, podremos cambiarlas por otras facilitadoras, que potencien emociones que favorezcan la acción (Sans, 2012). Generaremos, así, aprendizajes que empoderen al cuidador principal para conseguir sus propios objetivos.

De ahi la importancia de conocer esos filtros mentales y el uso que cada uno hace de ellos a la hora de interpretar su realidad. Si el profesional es capaz de identificar esos filtros en el adulto, tendrá a su disposición una herramienta más para adaptar su lenguaje durante la intervención, para que el cuidador principal con el que trabaja perciba que lo comprende y es capaz de ver la situación desde su propia perspectiva. Será entonces cuando sienta que él y el profesional hablan "el mismo idioma" y ven la realidad desde la misma perspectiva, consiguiendo mayor motivación e implicación.

Para conocer estos filtros mentales podemos recurrir a la Programación Neurolingüística (PNL). Bórquez (2002) desglosa los tres términos que la definen: hace referencia a una serie de esquemas (de ahí el término "programación") que vamos construyendo, desde nuestro nacimiento, en nuestro Sistema Nervioso (por ello el término "neuro", que viene de "neurológico"). Estos esquemas dan significado a todo aquello que percibimos, lo cual va siendo estructurado y puesto de manifiesto a través del lenguaje. tanto verbal como no verbal (de ahí el término "lingüística"). La PNL es un modelo práctico que nos ofrece una forma de pensar y nos da las herramientas para identificar la estructura de una comunicación eficaz en diferentes ámbitos como el educativo, sanitario o terapéutico (de

\section{Luynes, 1995).}

Dentro de estos filtros mentales tenemos la generalización, la distorsión y la omisión, que limitan la visión del mundo e influyen en la toma de decisiones (Villa, 2014). Podemos entender estos filtros como automatismos perceptivocognitivos, puesto que actúan desde y sobre la percepción y cognición de nuestras experiencias, dando sentido y significado a nuestra realidad.

Villa (2014) también nos hace reflexionar sobre la imposibilidad de registrar en nuestra mente todo lo que percibimos, señalándonos una serie de filtros mentales llamados metaprogramas, que determinan cómo procesamos y comunicamos la información recibida. Estos metaprogramas serian:

- De acercamiento hacia el placer o alejamiento del dolor.

- De referencia interna o externa.

- Proactivo o reactivo.

- Basado en las semejanzas o las diferencias.

- De opciones o procedimientos.

- De tiempo pasado, presente o futuro.

- Global o de detalle.

Conocer los metaprogramas de otra persona nos permite conocer sus actitudes, emociones y expectativas. Una información sumamente valiosa para seleccionar los mensajes verbales y no verbales que nos ayuden en el diálogo y a transmitir, con mayor nitidez, nuestros puntos de vista (Villa, 2014). De ahí la importancia que le damos al conocimiento de todos estos filtros en AT.

Finalmente, no podemos olvidar mencionar las creencias y valores, puesto que juegan un papel fundamental, en tanto que se forman a partir de nuestras experiencias individuales y sociales y dirigen nuestro comportamiento. Roberts (1999) describe las creencias como aquello que consideramos verdadero en un momento concreto, dejando que guien nuestra percepción e interpretación de la realidad. En cuanto a los valores, los define como aquello importante que motiva nuestra vida en un contexto particular.

En la Figura 1 resumimos gráficamente los 
filtros mentales mencionados. A continuación, profundizaremos en cada uno de ellos, ofreciendo ejemplos que servan de guía para identificarlos a través del lenguaje. También ofreceremos posibles estrategias para abordar y favorecer la reflexión en AT, partiendo de ese mismo filtro.

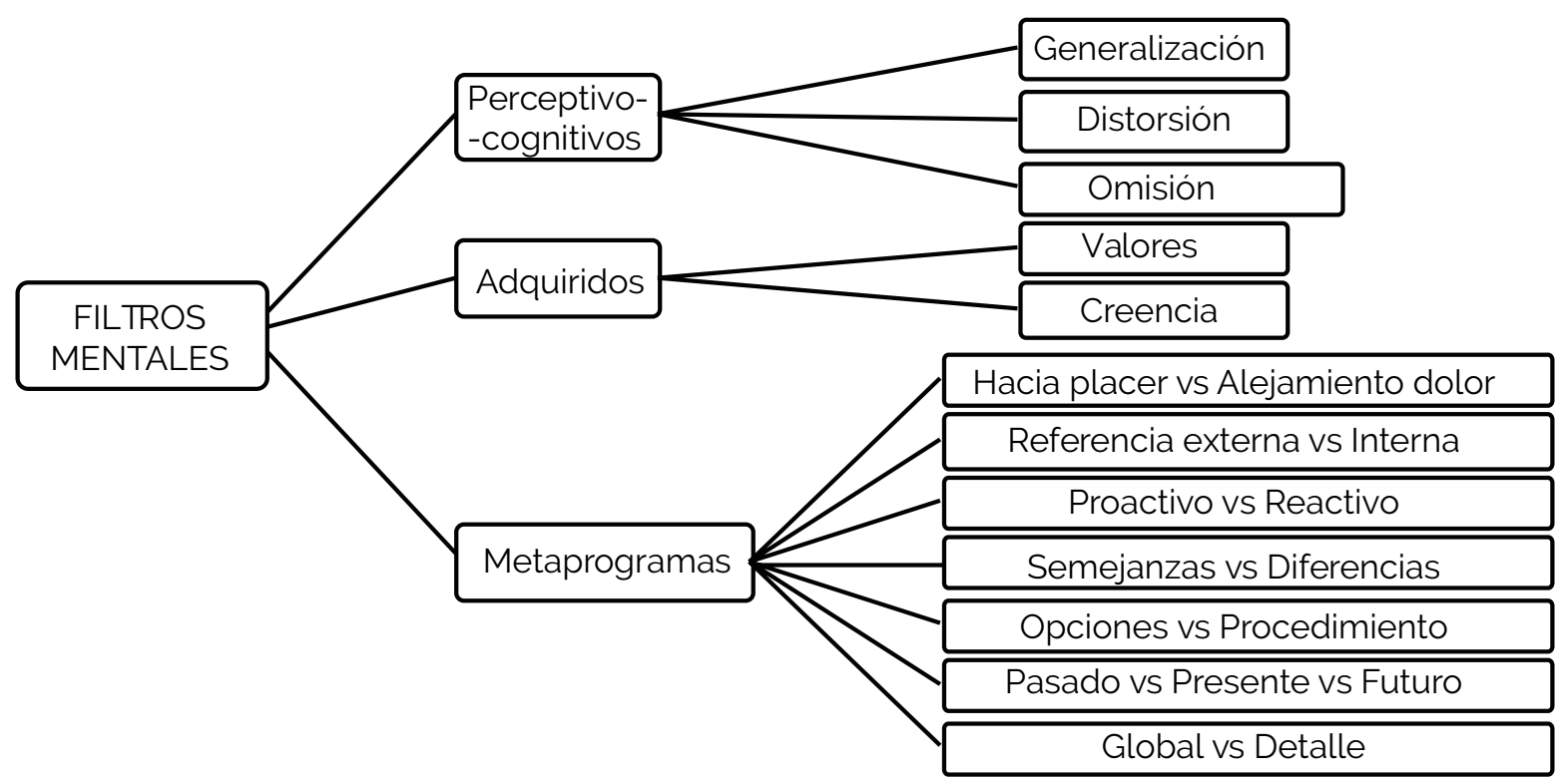

Figura 1 - Filtros mentales

Fuente: Preparado por los autores (2019)

\section{AUTOMATISMOS PERCEPTIVO-COGNITIVOS \\ GENERALIZACIÓN}

Se da cuando la persona convierte un fragmento de una experiencia vivida en una categoría completa (Castillo, 2007). Son personas que suelen utilizar expresiones como "todos", "ningún", "nadie", "siempre", etc. Ejemplos de este filtro mental son los siguientes comentarios de cuidadores principales: "No funciona nada"; "Lo he probado todo"; "no vemos ningún avance".

A la hora de responder a estos comentarios, el profesional puede partir de la propia generalización, para captar la confianza del cuidador y hacerle reflexionar sobre su postura y las implicaciones limitantes que puede tener la generalización para la toma de decisiones. Se pueden buscar ejemplos en los que se haya dado lo contrario a lo que comentan. También pude funcionar pedir algo más de concreción. Algunas preguntas que el profesional podría formular para favorecer la reflexión partiendo de la generalización, podrian ser:
- Cuando dices que no funciona nada, ¿a qué te refieres? ¿Qué cosas has intentado que no funcionan? ¿Las has probado siempre en el mismo momento y de la misma manera?

- ¿En qué situaciones has tenido la misma sensación otras veces, para otras metas que quisieras conseguir? ¿te has sorprendido en algún momento viendo que, a pesar de creer que no lo haria, si lo ha hecho?

- ¿Qué tiene que pasar para que veas un avance? Si repasamos los objetivos propuestos hasta ahora y valoramos su consecución, ¿siguen todos igual?

\section{DISTORSIÓN}

La distorsión es un proceso por el que transformamos la información que nos llega a través de nuestros sentidos (Blander \& Grinder, 2015: Bórquez, 2019). Según Castillo (2007), las distorsiones se dan cuando se intenta atribuir a otros la responsabilidad de nuestras acciones. En AT es frecuente encontrar distorsiones cuando la 
madre del niño con discapacidad se atribuye a sí misma la causa de dicha discapacidad, aunque esta fuera genética, por ejemplo. Un ejemplo sería el siguiente comentario de una mamá de una niña diagnosticada con una genopatía:

- Si hubiese acudido antes al hospital o al ginecólogo mi hija hubiese nacido sana. Cuando dejé de sentir sus patadas con la misma intensidad del principio debería haber acudido al médico.

Para responder ante una distorsión, el profesional puede solicitar pruebas de que eso es asi, preguntando por el hecho que lleva a tal afirmación. Puede invitar al cuidador a reflexionar sobre la utilidad de ese pensamiento. También es de utilidad poner ejemplos de situaciones similares previas, en las que haya habido una distorsión por parte del cuidador principal y se haya dado cuenta del error. Finalmente, puede invitar al cuidador a pensar otras formas de interpretar esa situación. Incluso plantearle qué le diría a una persona que tuviese ese mismo pensamiento. Por supuesto, junto a ello, facilitariamos toda la información necesaria para poder comprender la realidad (el diagnóstico y sus causas, en el ejemplo propuesto).

\section{OMISIÓN}

Se da cuando atendemos a determinados aspectos de nuestra experiencia y excluimos otros (Blander \& Grinder, 1975). Este filtro presenta un componente funcional ya que, realmente, no podemos atender a toda la información que nos llega. Por tanto, sólo nos quedamos con aquella información que, de alguna manera, nos es útil o llama nuestra atención, desechando el resto. El problema está cuando omitimos información clave para entender el problema. Un ejemplo de este proceso de omisión, en AT, seria:

- Pueden estar tranquilos que autista no es, no tiene autismo.

Ante este proceso, Bandler y Grinder (1975) proponen hacerle revivir, al cliente, aquella parte que no representó lingüisticamente. Insisten en que no merece la pena que el profesional presente alternativas a su cliente sobre aquello que omite, puesto que esas alternativas ya están eliminadas de su modelo mental y lo único que conseguiremos, es su rechazo. Más concretamente, se podrian hacer preguntas tales como "¿qué cosas tendrian que haber pasado para que no pensaras asi?". Incluso, valorar conjuntamente si, realmente, no se da ninguna de ellas. También, se podría proponer al cliente intentar llegar a una mayor concreción de la situación e, incluso, preguntar sobre quién, qué y cómo.

En cuanto a las posibles respuestas que puede dar el profesional, ante el comentario puesto como ejemplo, podemos proponer:

- ¿Qué piensas que puedo estar viendo en él que me hace pensar en autismo? ¿Qué caracteristicas? ¿De todas esas características, ¿cuáles son las que NO ves tú?

- ¿Puedes poner algún ejemplo que apoye lo que me dices?

- ¿Has hablado esto con tu pareja o con otro miembro de tu familia? ¿Y piensa igual o diferente a ti?

\section{METAPROGRAMAS}

Brown (2004) define los metaprogramas como un modelo de preferencias de personalidad que influyen en el lenguaje. Debemos tener claro que un metaprograma no define la personalidad, sino la forma de pensar y procesar la información (Lawley, 1997; Beddoes-Jones, 1999).

A continuación, describiremos cada uno de los metaprogramas identificados, sabiendo que todos ellos se describen en un continuo entre dos extremos y que, una misma persona, puede tener una preferencia por uno de esos extremos del continuo o combinar ambas preferencias (Brown, 2004). Además, en la Tabla 1 recogemos un listado de palabras que ayudan a identificar los dos extremos de algunos de estos metaprogramas (adaptado de Castillo, 2007). 
TABLA 1 - Expresiones que identifican los dos extremos de diferentes metaprogramas

METAPROGRAMA “HACIA EL PLACER" VS. “ALEJAMIENTO DEL DOLOR”

HACIA EL PLACER

ALEJAMIENTO DEL DOLOR

\begin{tabular}{cccc} 
Llevar a cabo & Lograr & Evitar & Solución \\
Conseguir & Alcanzar & Prescindir & Escapar \\
Obtener & Incluir & Prevenir & Remediar \\
Tener & & Deshacerse de... & \\
\hline
\end{tabular}

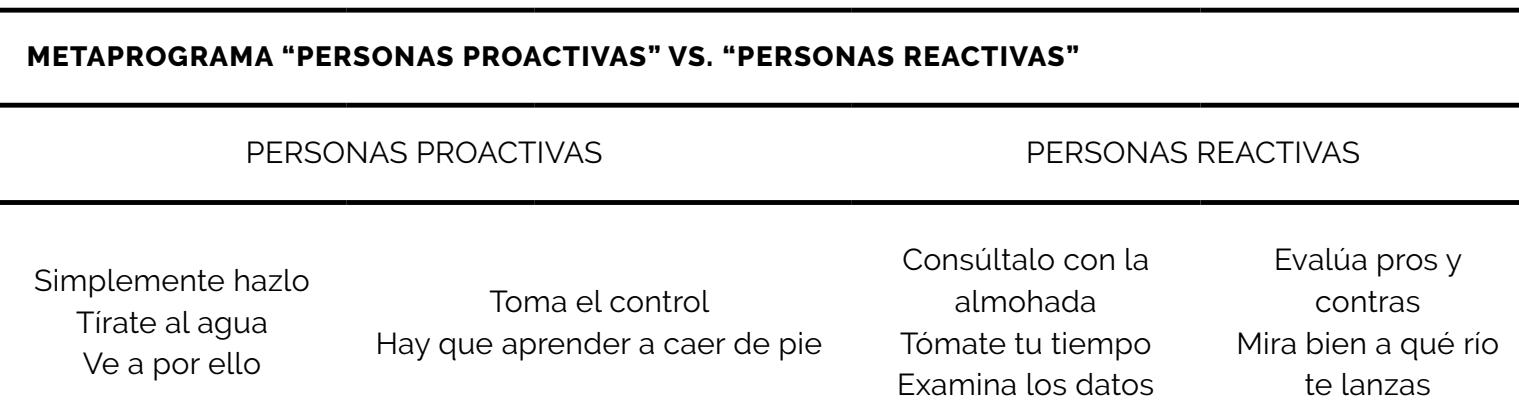

METAPROGRAMA “BÚSQUEDA SEMEJANZAS" VS “BÚSQUEDA DIFERENCIAS”

SEMEJANZAS

DIFERENCIAS

$\begin{array}{cccc}\text { Lo mismo } & \text { Estático } & \text { Como noche y día } & \text { No tiene } \\ \text { Igual } & \text { Tan bueno como... } & \text { Diferente } & \text { comparación } \\ \text { En común } & \text { Idéntico } & \text { Modificado } & \text { Completamente } \\ \text { Como siempre } & & \text { Cambiado } & \text { nuevo }\end{array}$

METAPROGRAMA “OPCIONES" VS “PROCEDIMIENTOS”

OPCIONES

PROCEDIMIENTOS

Ya veré llegado el momento

Hay que aprender a jugar con las normas
Juguemos con esa posibilidad Intenta lo mismo de otra forma
Sigue los pasos indicados

Respeta las normas

paso a paso
Primero

Segundo

Finalmente

Fuente: Preparado por los autores (2019)

Para ayudar a su comprensión, una vez más iremos proponiendo ejemplos que nos ayuden a entenderlos en AT. El conocimiento de los metaprogramas es una herramienta que nos puede servir para entender los patrones de nuestro interlocutor, lo que nos permitirá responderle con un lenguaje que entenderá fácilmente, mejorando la comunicación y facilitando la compenetración (Ready \& Burton, 2008)

\section{HACIA EL PLACER vS ALEJAMIENTO DEL DOLOR}

Las personas que se dirigen hacia el placer son aquellas que les motiva una meta y se dirigen hacia lo positivo. Por el contrario, las personas que tienden a alejarse del dolor suelen pensar en aquello que puede salir mal (Ready \& Burton, 2008).

Ante una persona enfocada hacia el placer, el profesional puede optar por darle mensajes positivos y hacerle reflexionar sobre los beneficios 
de marcarse un objetivo o poner en marcha una determinada estrategia. Por el contrario, ante una persona que presenta un metaprograma de alejamiento del dolor, el profesional puede hacerle reflexionar sobre la pérdida que puede suponer actuar o no de una determinada manera.

Un ejemplo de metaprograma enfocado hacia el placer, en AT, seria:

- Pero mi hijo, ¿cuándo va a hablar?

Algunas preguntas que puede realizar el profesional, podrian ser:

- De momento, ¿Qué cosas ves que lo están acercando al desarrollo del habla?

- ¿Qué va a suponer para ti que hable? Antes de llegar al habla, ¿te serviria comunicarte con tu hijo por otras vías, de otra manera?

- ¿Qué habilidades tiene tu hijo que le acercan a esa finalidad que has nombrado y para la que quieres que hable?

Un ejemplo de metaprograma de alejamiento del dolor, sería el comentario:

- Aquí te quedas tú con mi niño porque a mí me supera, he tirado la toalla.

El profesional puede abordar el comentario con preguntas como:

- ¿Qué significa que te supera?

- ¿Y qué puede pasar si...?

- ¿Qué cosas vas a perder si tiras la toalla?

- ¿Cuántas oportunidades de aprendizaje le voy a ofrecer yo teniendo en cuenta el tiempo que puedo compartir con él? y ¿cómo pueden ser esos momentos?

\section{REFERENCIA EXTERNA vS REFERENCIA INTERNA}

Las personas que tienen un marco de referencia externo son aquellas que ponen más atención en lo que piensan los demás y se dejan influir por ellos. Por su parte, las personas con una referencia interna son las que confian en sus propios instintos y no necesitan una aprobación externa. Estos conceptos reflejan las definiciones psicológicas clásicas de locus de control externo e interno (Rotter, 1966). Castillo (2007) nos dice que las personas con un metaprograma de referencia interna son personas seguras y con buena autoestima, mientras que las personas con un metaprograma de referencia externa son inseguras y tímidas.

Según Ready y Burton (2008), las personas con una referencia interna pueden ser algo más dificiles a la hora de hacerles entender el punto de vista de su interlocutor. Por su parte, las personas con una referencia externa pueden ser más fáciles de llevar, a pesar de necesitar del elogio y la dirección de sus superiores o de aquellos que ellos consideran con más poder y/o sabiduría que ellos mismos.

En cuanto a la forma de abordar la intervención en AT con cuidadores principales que presentan alguno de estos metaprogramas, podemos extrapolar las propuestas que nos hacen Ready y Burton (2008). Cuando se trate de una persona con una referencia interna pueden ser de utilidad frases como "tú eres el mejor juez", "lo dejo en tus manos", "mira a ver cómo lo haces", "examina los hechos y luego decides". Por el contrario, si se trata de un adulto con una referencia externa, las frases que se aconsejaría utilizar serian del estilo "la investigación dice...", "la opinión de los expertos dice que...", "esto tiene mucha aceptación".

Dentro del ámbito de la AT, podemos tomar como ejemplo un comentario muy utilizado por muchas familias, en el que queda reflejada la influencia externa:

- El neuropediatra dice que necesita más sesiones.

Algunas preguntas para abordar dicho comentario serían:

- ¿Qué sabe el neuropediatra de la forma en que estamos trabajando?

- Desde que se levanta tu niño hasta que se acuesta, ¿cuántas veces intentas conseguir el objetivo marcado?

- Si el neuropediatra supiera exactamente cómo trabajamos y las oportunidades de aprendizaje que tú como mamá o papá estás ofreciendo a tu niño, ¿en cuántas 
sesiones crees que él fijaría el trabajo que estamos haciendo con el niño?

El siguiente comentario refleja la influencia de la referencia interna:

- Todo esto está muy bien, pero yo necesito un logopeda que trabaje con mi hija.

A continuación, recogemos posibles preguntas para abordar dicho comentario:

- ¿Y qué necesitas del logopeda?

- ¿Qué piensas que te va a aportar el logopeda?

- ¿Qué va a conseguir el logopeda que no puedes conseguir tú?

\section{PROACTIVO vs REACTIVO}

Las personas proactivas son aquellas que se hacen cargo de la situación y actúan. Es decir, son personas capaces de dar solución a situaciones urgentes (Ready \& Burton, 2008). Numerosos autores describen a las personas proactivas como aquellas con conductas autónomas, intrinsecamente motivadas, orientadas al cambio y centradas en el futuro (Belschak \& Den Hartog, 2010; Grant \& Ashford, 2008; Parker \& Collins, 2010; Salessi \& Omar, 2017; Tornau \& Frese, 2012). Por su parte, las personas reactivas son las que dejan que otros tomen las riendas de la situación, tomando la iniciativa sólo cuando consideran que es el momento oportuno y tras ver cuáles son las consecuencias de actuar de una manera u otra (Ready \& Burton, 2008). En la Tabla 1 resumimos un listado de palabras y expresiones que suelen utilizar las personas proactivas y reactivas.

En términos generales, la forma de abordar la intervención, tanto con personas proactivas como reactivas, se basará en proponer situaciones similares y hacerles reflexionar sobre cómo actuarian. También en reflexionar sobre la forma de actuar proactiva o reactivamente, qué consecuencias tiene o puede tener y si esas consecuencias les aportan algo positivo. En cualquier caso, siempre sería aconsejable poner ejemplos que hayan ocurrido y analizar las consecuencias.
Un ejemplo de comentario que incluiríamos en el metaprograma proactivo sería:

- Me han dicho lo que debo hacer cuando se muerde, pero no cómo evitar que se lleve la mano a la boca. Sería mejor evitarlo desde el principio, no cuando ya se ha mordido.

Preguntas que el profesional puede realizar para este metaprograma serian:

- ¿Por qué crees que es mejor evitar que se lleve la mano a la boca y no actuar cuando ya empieza a morderse?

- ¿Te has fijado en lo que está pasando cuando no se mete la mano a la boca? ¿Por qué crees que no lo hace en esos momentos?

- ¿Cómo crees que podrías evitar que se lleve la mano a la boca?

En el extremo opuesto de este metaprograma tendriamos el siguiente comentario que se corresponde a un adulto reactivo:

- Aunque le quite lo que tiene a su alcance, siempre da con otra cosa para lanzarla. Asi que "acción-reacción", cuando lo lanza le hago recogerlo.

Para el trabajo con este cuidador, el profesional puede formular preguntas como:

- ¿Te sientes cómodo reaccionando así?

- ¿Qué piensas que está aprendiendo tu hijo ante esta situación? ¿Qué te gustaría que aprendiese?

- ¿Qué conducta es la que está repitiendo? ¿Qué piensas que le puede aportar?

- ¿Te gustaría evitar que lance los objetos? Cuando tú reaccionas una vez que ya los ha lanzado, ¿estás evitando que eso suceda en próximas ocasiones? ¿Cómo crees que podrias evitarlo?

\section{BÚSQUEDA DE SEMEJANZAS vs BÚSQUEDA DE DIFERENCIAS}

Las personas que buscan las semejanzas intentan cotejar la información nueva con la que 
ya conocen, mientras que ignoran la información que es diferente. Todo lo contrario pasa con las personas que buscan las diferencias, puesto que se fijan en aquello que cambia e ignoran las similitudes (Ready \& Burton, 2008). En la Tabla 1 resumimos ejemplos de palabras o expresiones que suelen emplear personas que buscan las semejanzas y las que buscan las diferencias.

El profesional de AT, que lleva a cabo unas PCF, puede favorecer la reflexión del adulto enfocado en las semejanzas o las diferencias, utilizando estas mismas palabras durante la intervención, con el fin de promover una mayor confianza y empatía entre ellos. Por otra parte, también pude hacerle reflexionar sobre los aspectos similares o diferentes, según el metaprograma dominante. que pueden tener otras situaciones con respecto a la que está contando.

El siguiente comentario ejemplifica un metaprograma enfocado en la semejanza:

- Llevamos mucho tiempo hablando, pero vemos que el niño no avanza.

En este caso el profesional puede plantearle preguntas del tipo:

- Desde que empezamos a hablar hasta ahora, ¿qué cosas siguen igual?

- ¿Cuánto tiempo llevamos hablando? ¿Cuánto tiempo crees que necesitaría para conseguir que avance en ese objetivo?

Por otra parte, también se puede analizar con él la relación de objetivos que se ha planteado este cuidador principal desde que empezó en AT o desde un momento concreto y valorar su consecución, haciéndole reflexionar sobre si esos objetivos siguen todos igual y a qué cree que se debe.

Un ejemplo de comentario enfocado en las diferencias sería:

- Sólo habla contigo. Yo no sé qué hacer para que hable conmigo.

Ante este comentario, el profesional puede llevarlo a reflexionar sobre las diferencias concretas entre uno y otro, ya que verbalizar y concretar esas diferencias, puede llevarle a adoptar otras medidas o estrategias para conseguir sus objetivos.

\section{DE OPCIONES vS DE PROCEDIMIENTOS}

Las personas con un metaprograma de opciones necesitan variedad y cambios constantes. En cambio, las personas con un metaprograma de procedimientos prefieren ceñirse a unas normas establecidas y, posiblemente, las sigan de forma sistemática y sin modificaciones (Ready \& Burton, 2008). En la Tabla 1 se muestran ejemplos de expresiones que suelen utilizar las personas con predominio de estos metaprogramas.

A la hora de trabajar con un adulto cuyo metaprograma predominante es de opciones, el profesional podría ofrecerle varias opciones de actuación y pedirle que elija la que mejor pueda llevar a cabo. Además, convendría dejarle claro que la elección de una opción no significa que no pueda probar las otras en otro momento. También, se debería hacer reflexionar al adulto sobre las ventajas y desventajas de esa opción.

En cuanto a los adultos que presentan un metaprograma de procedimientos, el profesional podria ofrecerles procedimientos a seguir y ayudarles a secuenciarlos, así como analizar en qué punto se encuentran y cuál es el siguiente paso.

Ejemplo de un comentario donde predomina el metaprograma de opciones:

- Claro, esta opción es mucho más fácil porque no tenemos que ponernos a trabajar, sino que lo hacemos todos los días.

A este comentario, el profesional puede preguntar:

- Ahora...¿qué opciones te ofrece el día para...(conseguir el objetivo marcado)?

- ¿En qué momentos te puedes plantear conseguirlo? ¿Y cómo? ¿Qué posibilidades tienes para hacerlo?

- ¿Qué objetivos crees que puedes conseguir al cabo del día?

Ejemplo de un comentario donde predomina el metaprograma procedimientos: 
- Muchas gracias, ahora sé lo que tengo que hacer y los pasos que tengo que seguir.

En este caso, el profesional simplemente deberia ayudarle a concretar aquello que debe hacer y los pasos que debe dar, para ello puede utilizar expresiones como:

- Ok, vamos a poner nombre y especificar lo que tienes que hacer.

- ¿Cuál es tu objetivo para esta semana? $Y$, ¿qué vas a hacer para conseguirlo?

\section{TIEMPO PASADO vS TIEMPO PRESENTE vS TIEMPO FUTURO}

Es un metaprograma fácil de identificar a través del lenguaje, puesto que podemos reconocerlo prestando atención al tiempo verbal que el cuidador principal utiliza mayoritariamente en su discurso. Una persona que suele hacer un mayor uso del tiempo pasado, será una persona más enfocada en el pasado, mientras que las personas enfocadas en el presente utilizan el tiempo verbal presente y las personas enfocadas en el futuro suelen utilizar dicho tiempo verbal en sus conversaciones.

A la hora de trabajar con el adulto en función del metaprograma referido al tiempo, aconsejaríamos que, para aquellas personas enfocadas en el pasado, el profesional recupere situaciones similares del pasado que desmonten o reafirmen la situación en cuestión, asi como utilizar verbos en pasado. Para aquellas personas enfocadas en el presente, la recomendación sería utilizar ese tiempo verbal. Finalmente, para las personas enfocadas en el futuro, se recomienda utilizar verbos en futuro, ayudarlas a proyectarse en el futuro y hacerlas reflexionar sobre cómo les gustaria verse, incluso preguntarles qué les gustaria haber conseguido dentro de 5 ó 6 meses.

\section{GLOBAL vs DETALLE}

Las personas con un metaprograma global son aquellas que atienden a la idea general, pudiendo desconectar o impacientarse ante la mayor cantidad de información que pueden ofrecer y en la que pueden centrarse las personas con un metaprograma basado en el detalle (Ready \& Burton, 2008). Las personas con un metaprograma global pueden saltarse algún paso cuando describen secuencias o situaciones. Por el contrario, las personas con un metaprograma de detalle hablarán de pasos y secuencias, haciendo descripciones más precisas (O'Connor \& Seymour, 2007).

A la hora de trabajar con personas con un metaprograma global, Ready y Burton (2008) aconsejan utilizar términos como: "perspectiva general", "en dos palabras", "por lo general" o "esencialmente". En cuanto a las personas con un metaprograma basado en el detalle, entenderán mejor el mensaje con expresiones como: "plan", "precisamente", "de manera específica", "primero", "segundo", etc.

Un comentario ejemplo de un metaprograma global seria:

- Hace las cosas cuando quiere.

Ante este comentario, el profesional puede formular preguntas como:

- ¿Me estás diciendo que hay veces que si lo hace?

- ¿Cómo podríamos intentar que quiera siempre?

- ¿Qué significa "cuando quiere"?

- ¿Cuándo quiere?

Un ejemplo del metaprograma enfocado en el detalle, en AT, sería el siguiente:

- Sólo cuando nosotros sostenemos el bibe es capaz de tomárselo. Nosotros se lo dejamos encima de la mesa y le decimos que lo coja. Pero él se limita a sentarse en el sofá y esperar a que se lo llevemos. Al final, acabamos cogiéndolo nosotros y dándoselo. Cuando ya se lo está tomando intentamos que sea él quien lo coja y, cuando creemos que lo ha hecho, intentamos soltarlo poco a poco, a ver si así lo sostiene él, pero que va, en el momento que nota que lo soltamos nosotros, él lo suelta...

Como vemos, las personas con este metaprograma tenderán a dar mucha información, 
aún sin ser necesaria. La tarea del profesional, en este caso, consistirá en hacerlos ver y reflexionar sobre los detalles realmente útiles y necesarios para seguir avanzando en los objetivos que la familia se marque. Nos podría servir hacerles reflexionar sobre el tipo de biberón que utiliza el niño y si, este, favorece su agarre. Para ello se podrian utilizar preguntas del tipo:

- En toda esa situación que habéis descrito, ¿qué pensáis que está dificultando el que el niño coja el bibe por sí solo?

- Cuando el niño coge el bibe, ¿cómo lo coge?

- ¿Cómo es el bibe que le dais?

- ¿Creéis que puede pesar mucho para que el niño lo agarre cuando lo llenáis?

- ¿El bibe tiene asas para facilitar que el niño lo agarre?

Además, en este caso también podria ser útil ver al niño en situación, en el momento de tomar el biberón, o bien a través de alguna grabación que se pudiese analizar posteriormente con la familia.

\section{FILTROS ADQUIRIDOS: CREENCIAS Y VALORES}

Mención especial merecen las creencias y valores de la persona. Cada uno de nosotros actuamos en base a creencias y valores que pueden diferir tanto de unos a otros, como de un momento a otro para la misma persona. Estas creencias y valores marcarán nuestra actitud y nuestras acciones frente a la vida. Pero también pueden cambiar, en uno mismo, en función de la etapa por la que estemos atravesando. Igualmente, actuarán como filtros a la hora de interpretar la realidad que nos rodea. Para O'Connor $Y$ Seymour (2007), las creencias y valores son los que configuran nuestras vidas y le dan sentido.

Según Dilts (2003), las creencias nos permiten dar significado a nuestras experiencias, favoreciendo la generalización y contribuyendo a poner los límites a nuestro mundo, nuestro comportamiento y nuestras capacidades. De ahí que Simón (2016) nos haga reflexionar sobre la posibilidad de cambiar las creencias, afirmando que un cambio de creencias puede provocar un gran cambio en la forma en que vivimos nuestras vidas y en la manifestación de nuestros comportamientos.

En cuanto a los valores, según O'Connor y Seymour (2007) están apoyados en las creencias $y$, al igual que estas, los adquirimos a través de nuestras propias experiencias con la vida, siendo considerados nuestra seña de identidad y los principios que rigen nuestra forma de vivir.

Aceptada la importancia de conocer todos los filtros mentales del coachee que, en nuestro caso, se corresponde con el cuidador principal del niño con necesidades de AT, debemos recordar que entre esos filtros se encuentran las creencias y los valores. Al igual que se recomienda utilizar el mismo lenguaje que utiliza el coachee, una vez que se ha identificado cualquiera de los filtros mencionados hasta ahora, también es recomendable indagar sobre las creencias y valores de este adulto, con el fin de respetarlos y basar la intervención en aquello que da sentido a su forma de vivir.

Tal y como nos dicen O'Connor y Seymour (2007), compartir los valores de otra persona ayudará a crear una buena sintonía con ella. $Y$ eso es lo que el profesional de AT necesita como punto de partida para favorecer la confianza de ese adulto con el que trabaja y, a partir de ahí, hacerlo competente en el desarrollo de su hijo y su familia. No podemos tener las mismas creencias y valores de todos los cuidadores principales con los que trabajamos, pero si podemos mostrar, explícita e implícitamente, un total respeto hacia ellos.

\section{CONCLUSIONES}

Tal y como hemos ido señalando a lo largo del texto, con frecuencia las personas pueden modificar sus estrategias y filtros mentales, algo que facilitaremos si hablamos con ellos en su propio lenguaje. Esta afirmación nos ha de servir para apoyar la necesidad de conocer esos filtros y las expresiones con las que se manifiestan, con el fin de poder utilizar el mismo lenguaje que utiliza el coachee o cuidador principal. Utilizando ese 
mismo lenguaje, podremos hacerle entender, al adulto con el que trabajamos, que vemos la realidad con sus mismos filtros y, con ello, estaremos haciendo nuestra comunicación más fácil y efectiva. Tal y como nos dice Brown (2004), la caracteristica fundamental de cualquier relación entre dos o más personas que se comunican es la sintonía que se crea entre ellos, enfocándose en la misma información. Y esa focalización en la información que comparten cuidador principaly profesional de AT, es fundamental para favorecer la autocompetencia del adulto y, a partir de ahi, la mejora en el desarrollo de su hijo.

A lo largo de todo este artículo hemos facilitado una serie de orientaciones sobre el tipo de preguntas o comentarios que pueden hacer, tanto el cuidador principal como el profesional que trabaja con él, dependiendo del filtro que este identifique en el adulto con el que trabaja. Es importante entender que no son más que ejemplos didácticos. El tipo de preguntas o comentarios del profesional es algo personal, que viene influido también por sus propios filtros mentales. Lo importante es entender que deberiamos ser conscientes de los filtros del adulto con el que trabajamos y abordar las situaciones en base a los filtros del coachee, para favorecer esa comunicación.

De la misma manera, tenemos que saber que los diferentes filtros mentales mencionados aquí no son excluyentes entre sí. Cada uno puede operar en la misma persona en momentos distintos. No obstante, si es cierto que en cada persona predomina un tipo de filtro frente a otro.

Tampoco podemos olvidar, el papel fundamental que juegan las emociones a la hora de interpretar la realidad y tomar decisiones. Por ello, independientemente del metaprograma que predomine en la persona, también se aconseja siempre conectar con las emociones que puede generar o haya generado una determinada situación.

Con todo lo dicho, es cierto que identificar un determinado filtro en el adulto no es tarea fácil. Además, es muy importante que, ante un proceso de intervención en el que vamos a utilizar el coaching y la PNL como una de sus herramientas esenciales, lo primero que debemos hacer es asegurarnos del filtro mental que utiliza nuestro coachee. No son extraños aquellos casos en los que, inicialmente, el coach identifica un determinado filtro, pero a lo largo de la conversación se da cuenta de que, realmente, el filtro predominante es otro. Con un poco de entrenamiento, y siempre a través del diálogo y la escucha activa, el profesional irá adquiriendo la habilidad necesaria.

Por tanto, como profesionales de AT que queremos llevar a cabo una PCF, no deberiamos desperdiciar esta oportunidad de aprendizaje, tanto más cuando nos puede ayudar en nuestra labor con esas familias. Sobre todo si, como profesionales de la AT, buscamos favorecer la reflexión en los cuidadores principales del niño, para que ganen competencias en su labor de padres y puedan tomar aquellas decisiones que mejor cubran sus necesidades y atiendan sus prioridades.

\section{REFERENCIAS BIBLIOGRÁFICAS}

Bandler, R., \& Grinder, J. (1975). La estructura de la magia I. [S.L.]: Diegoan. Recuperado Agosto 7, 2019. de: https://cdn.hermandadblanca.org/wp-content/ uploads/2018/06/la-estructura-de-la-magia-i-richard-bandler.pdf

Beddoes-Jones, F. (1999). Thinking styles, relationship strategies that work! Lincolnshire: BJA Associates Ltd.

Belschak, F. D., \& Den Hartog, D. N. (2010). Pro-self, prosocial, and pro organizational foci of proactive behaviour: Differential antecedents and consequences. Journal of Occupational and Organizational Psychology, 83(2), 475-498. https://doi. org/10.1348/096317909×439208

Benavides, S. (2014). El coaching en el ámbito empresarial. (Trabajo Fin de Grado-Facultad de Educación). Illes Balears: Universitat.

Bisquerra, R. (2008, cuatrimestre $2^{\circ}$ ). Coaching: un reto para los orientadores. Revista Española de Orientación y Psicopedagogía, 19(2), 163-170.

Bórquez, S. (2002, mayo - junho). PNL: tres letras para facilitar el cambio. Pharos, 9(1). Recuperado Agosto 5, 2019, de: http://www.redalyc.org/ $\mathrm{pdf} / 208 / 20809106 . \mathrm{pdf}$.

Brown, N. (2004, September). What makes a good educator? The relevance of meta programmes. Assessment and Evaluation in Higher Education, 29(5), 515533. https://doi.org/10.1080/0260293042000197618 
Castillo, A. (2007). Actitudes y habilidades del terapeuta. Cultura, 21, 211-227. Recuperado Agosto 5 2019, de: http://wwww.revistacultura.com.pe/revistas/ RCU_21_1_actitudes-y-habilidades-del-terapeuta.pdf

De Luynes, M. (1995). Neuro linguistic programming. Educational and Child Psychology, 12(4), p. 34-47.

Dilts, R. (2003). El poder de la palabra. Barcelona: Urano.

Dunst, C. J. (2000, April). Revisiting "Rethinking Early Intervention." Topics in Early Childhood Special Education, 20(2), 95-104. https://doi. org/10.1177/027112140002000205

Dunst, C. J. (2002). Family-Centered Practices: Birth through High School. The Journal of Special Education, 36(3), 139-147. https://doi.org/10.1177/00224669 020360030401

Espindola, J. L. (2005). Análisis de problemas y toma de decisiones. Mexico: Pearson Educación.

Friedman, M., Woods, J., \& Salisbury, C. (2012, enero/ marzo). Caregiver Coaching Strategies for Early Intervention Providers. Infants and Young Children. Philadelphia, 25(1), 62-82. https://doi.org/10.1097/ iyc.0b013e31823d8f12

Grant, A. M.; Ashford, S. J. (2008). The dynamics of proactivity at work. Research in Organizational Behavior, 28, 3-34. https://doi.org/10.1016/j. riob.2008.04.002

Guarnieri, S., \& Ortiz, M. No es lo mismo. Madrid: Editorial LID, 2010.

Lawley, J. (1997). The application of meta programmes in the classroom. Rapport, 37, 7-11.

Lozano, L. J. (2008, mayo/agosto). El coaching como estrategia para la formación de competencias profesionales. Revista Escuela de Administración de Negocios, 63. 127-137. https://doi.org/10.21158/01208160.n63.2008.447

O'Connor, J., \& Seymour, J. (2007). Introducción a la Programación Neurolingüistica. Barcelona: Urano.

Parker, S. K., \& Collins, C. (2010 mayo). Taking stock: Integrating and differentiating multiple proactive behaviors. Journal of Management, 36(3), 633-662. https://doi.org/10.1177/0149206308321554

Ready, R., \& Burton, K. (2008). PNL para Dummies. Barcelona: Granica.

Roberts, M. (1999). Change Management Excellence: Putting NLP to work. Wales: Crown House Publishing Ltd.

Rush, D., \& Shelden, M. L. (2011). The early childhood coaching handbook. Baltimore, MD: Brookes Publishing.

Rotter, J. B. (1996). Generalized expectancies for internal versus external control of reinforcement. Psychological Monographs: General and Applied. 80(1), 1-28. https://doi.org/10.1037/hoog2976

Salessi, S., \& Omar, A. (2017). Comportamientos proactivos en el trabajo: una puesta al dia. Revista argentina de ciencias del comportamiento, 9(3), 82-103. https://doi.org/10.15517/ap.v32i124.30642
Sans, M. (2012, Junio). ¿Qué es el coaching? Sus orígenes, definición, distintas metodologias y principios básicos de actuación de un coach. $3 \mathrm{C}$ Empresa: investigación y pensamiento crítico, 1(3), 28-37. Recuperado Julio 28, 2019, de: https://www. 3ciencias.com/wp-content/uploads/2012/06/3. Que-es-Coaching.pdf https://doi.org/10.17993/ 3cemp.2016.050125.47-56

Simón, C. (2016). Identifica y cambia tus creencias limitantes. [S.L.]: Mindful Science, Recuperado Agosto 10, 2019, de: https://www.mindfulscience.es/wp-content/uploads/2016/12/ ebook-identifica-y-cambia-tus-creencias-limitantes.pdf

Tornau, K., \& Frese, M. (2012). Construct clean-up in proactivity research: A meta analysis on the nomological net of work-related proactivity concepts and their incremental validities. Applied Psychology: An International Review, 62(1), 44-96. https://doi. org/10.1111/j.1464-0597.2012.00514.X

Villa, M. E. (2014). Programación neurolingüística. Concepto y aplicaciones en la Organización. Revista QUESTIONAR: Investigación Específica, 1(2), 31-42.

\section{Noelia Orcajada Sánchez}

Licenciada en Pedagogía y Diplomada en Logopedia por la Universidad de Murcia. Doctorado en Educación por la Universidad de Murcia. Profesora en el Dpto. MIDE, Facultad de Educación de la misma Universidad. Directora de un gabinete psicopedagógico y logopédico y directora y coordinadora de un equipo de Atención Temprana centrado en la Familia. Regularmente ofrece apoyo a diferentes profesionales en proceso de transformación hacia una prácticas centradas en la familia.

\section{Francisco Alberto García Sánchez}

Catedrático, Dpto. MIDE, coordinador del Grupo de Investigación en Educación, Diversidad y Calidad (Universidad de Murcia). Doctor en Psicologia por esta Universidad. Coordinador del Dpto. de Documentación, Investigación y Desarrollo de ASTRAPACE, forma parte de su equipo de dirección del Servicio de Atención Temprana (AT) centrado en la familia. Co-atuor del Libro Blanco de la AT y decenas de artículos y capítulos de libros sobre Atención Temprana. Ponente habitual en Reuniones Científica, Masters y Cursos de Formación y Especialización sobre esta disciplina. Participación en ocho proyectos del Plan Nacional I+D. Reconocidos 4 sexenios de investigación

\section{Endereço para correspondencia:}

Facultad de Educación

Campus Universitario de Espinardo

30100 Murcia, Espanha 